\title{
Osteoblast Differentiation Stage-Specific Expression of the Pyrophosphate-Generating Enzyme PC-1
}

\author{
Nan E. Hatch Renny T. Franceschi \\ Department of Orthodontics and Pediatric Dentistry, School of Dentistry, University of Michigan, \\ Ann Arbor, Mich., USA
}

\section{Key Words}

Fibroblast growth factor - PC-1 - Pyrophosphate •

Osteoblast $\cdot$ Mineralization

\begin{abstract}
Fibroblast growth factor (FGF) signaling plays a critical role in skeletal development, yet the mechanism by which FGFs affect bone mineralization is not well understood. Review of the literature investigating effects of FGF signaling on bone mineralization indicates that FGFs may stimulate expression of factors that prevent mineralization in the short term and enhance mineralization in the long term. Pyrophosphate is an ideal example of a factor that, dependent upon environment, has the capacity to inhibit or enhance mineralization. PC-1 is the primary generator of pyrophosphate in osteoblastic cells; therefore, regulated expression of PC-1 by FGFs may be a principal mechanism by which FGF signaling affects bone mineralization. We previously showed that FGF2 induces PC-1 expression in preosteoblastic cells and that this induction is differentiation stage dependent. In order to more directly investigate the mechanism by which PC-1 expression is regulated, we have cloned a $2.8-\mathrm{kb}$ region of the PC-1 gene promoter and constructed a PC-1 gene promoter/ firefly luciferase reporter construct. Results indicate that this construct is specifically responsive to FGF2 or ascorbate (an inducer of osteoblast differentiation). Promoter responsiveness to FGF2 is significantly diminished upon osteoblast differentiation, and increases in promoter activity that occur
\end{abstract}

with osteoblast differentiation are inhibited by FGF2 treatment. These results indicate that the mechanism of PC-1 induction by FGF2 in preosteoblastic cells is distinct from the mechanism of induction that occurs with osteoblast differentiation. These results also indicate that PC-1 may play multiple and distinct roles in the development of mineralized tissues.

Copyright $\odot 2008$ S. Karger AG, Base

\section{Introduction}

Close review of the literature investigating the effects of fibroblast growth factors (FGFs) on bone mineralization yields a complex and superficially paradoxical story. FGF2 knockout mice exhibit significantly diminished bone mass, bone formation rate and trabecular bone volume, suggesting that FGF2 is a positive regulator of bone formation, yet FGF2 overexpression in mice also results in significantly diminished bone density and trabecular bone volume [Montero et al., 2000; Sobue et al., 2005].

\section{Abbreviations used in this paper}

AP alkaline phosphatase

ATP adenosine triphosphate

FGF fibroblast growth factor

\section{KARGER}

Fax +4161306 1234

E-Mail karger@karger.ch

www.karger.com
(C) 2008 S. Karger AG, Basel

$1422-6405 / 09 / 1894-0065 \$ 26.00 / 0$

Accessible online at:

www.karger.com/cto
Dr. Nan Hatch

Department of Orthodontics and Pediatric Dentistry, School of Dentistry University of Michigan, 1011 N University Avenue

Ann Arbor, MI 48109-1078 (USA)

Tel. +1 734615 8792, Fax +1 734763 8100,E-Mail nhatch@umich.edu 
Similarly, bone marrow stromal cells from both FGF2 knockout and FGF2 overexpresser mice display a dramatically diminished ability to form mineralized nodules in culture. Furthermore, while treatment of mice with systemic FGF1 and FGF2 results in an overall bone anabolic affect, this effect does not occur until after a period of withdrawal following FGF treatment [Dunstan et al., 1999; Liang et al., 1999]. Taken together, these results indicate that the bone anabolic effects of FGFs include a transient inhibition of bone mineralization. These results also suggest that FGFs may act to stimulate expression of factors that prevent mineralization in the short term and enhance mineralization in the long term. Pyrophosphate is an ideal example of such a bifunctional factor because although pyrophosphate itself inhibits mineralization, pyrophosphate can also act to enhance mineralization when it is hydrolyzed to phosphate [Register and Wuthier, 1958; Anderson et al., 2005]. To date, the factors involved in the bone mineralization effects of FGF signaling are unknown. It is our hypothesis that the mineralization effects of FGFs can be explained by FGF changes in expression of factors that control the elaboration and hydrolysis of pyrophosphate.

PC-1 (ectonucleotide pyrophosphate/phosphodiesterase-1, ENPP1) is a nucleoside triphosphate pyrophosphohydrolase that generates pyrophosphate from the hydrolysis of adenosine triphosphate (ATP) [Terkeltaub et al., 1994]. PC-1 is expressed in differentiated osteoblasts and is known to be the primary enzymatic generator of pyrophosphate in osteoblastic cells [Johnson et al., 1999, 2001]. PC-1 activity increases extracellular levels of pyrophosphate, which inhibits hydroxyapatite crystal deposition and growth. Yet, this pyrophosphate also serves to enhance mineralization when it is hydrolyzed into phosphate by the osteoblastic enzyme alkaline phosphatase (AP). The critical role of PC-1-generated pyrophosphate in bone mineralization is evidenced by the fact that PC-1 null mice exhibit significantly diminished physiologic bone mineralization [Johnson et al., 2003]. Significantly, a cross of the $\mathrm{AP}^{-/-}$mouse with the $\mathrm{PC}-1^{-/-}$mouse generates a double knockout mouse with apparently normal bone mineralization [Hessle et al., 2002; Anderson et al., 2005]. This result supports the idea that PC-1 and AP work together to produce normally mineralized bone matrix via the elaboration and hydrolysis of pyrophosphate.

We previously showed that FGF2 specifically upregulates expression of PC-1, while inhibiting expression of AP, in calvarial preosteoblastic cells [Hatch et al., 2005]. This combined pattern of expression is expected to in- crease extracellular levels of pyrophosphate. Concordantly, FGF2 treatment inhibited mineralized nodule formation by these cells. Because the induction of PC-1 by FGF2 in preosteoblastic cells may be a primary mechanism by which FGFs affect bone mineralization, and because PC-1 expression is known to be critical for normal bone mineralization, we have initiated an investigation into the mechanism by which PC-1 expression is regulated in osteoblastic precursor cells and in differentiated osteoblasts. Here we confirm that PC-1 promoter activity increases with either osteoblast differentiation or FGF2 treatment. Additionally, we show that osteoblast differentiation diminishes promoter responsiveness to FGF2 and that FGF2 treatment diminishes promoter responsiveness to osteoblast differentiation. These results indicate that the mechanism of PC-1 induction in preosteoblastic cells is distinct from the mechanism of induction that occurs with osteoblast differentiation. They also indicate that PC-1 may play multiple and distinct roles in the development of mineralized tissues, and that the role of preosteoblastic PC-1 may not be the same as that for PC-1 in differentiated osteoblasts.

\section{Materials and Methods}

\section{Cell Culture}

MC3T3E1(C4) cells are a calvarial preosteoblastic cell line that has been shown to exhibit high osteoblastic differentiation potential upon addition of ascorbate. To maintain cells in an undifferentiated state, MC3T3E1(C4) cells were cultured in custom-formulated $\alpha$-MEM containing no ascorbate supplemented with $10 \%$ fetal bovine serum and penicillin/streptomycin. To induce differentiation, MC3T3E1(C4) cells were cultured in $\alpha$-MEM supplemented with $10 \%$ fetal bovine serum, penicillin/streptomycin and $50 \mu \mathrm{g} / \mathrm{ml}$ ascorbate. For all experiments, cells were plated at 300,000 cells per $60-\mathrm{mm}^{2}$ plate and grown at $37^{\circ} \mathrm{C}, 5 \% \mathrm{CO}_{2}$.

\section{DNA Constructs}

A 2,864-bp region of the proximal PC-1 gene promoter was cloned by PCR utilizing the primers 5'-AGAGCGGGACGATGGAGCGCGA-3' and 3'- CCTACCAGCGACAGCACTTTGTAGGTGT-5'. This PCR product was digested with the restriction enzymes Kpn1 and Xhol, purified, and ligated into PGL3basic vector (Promega) to create the PGL3/PC1 gene promoter/reporter construct. The translation initiation start site of the $\mathrm{PC}-1$ promoter was subsequently eliminated via in vitro mutagenesis utilizing the QuickChange Site Directed Mutagenesis Kit (Stratagene). The renilla luciferase construct PGL4/hRluc (Promega) was utilized as an internal control for transfection and/or cell lysis efficiency.

\section{Transfections}

Superfect transfection reagent (Qiagen) was utilized for all transfection experiments, following the manufacturer's recom- 


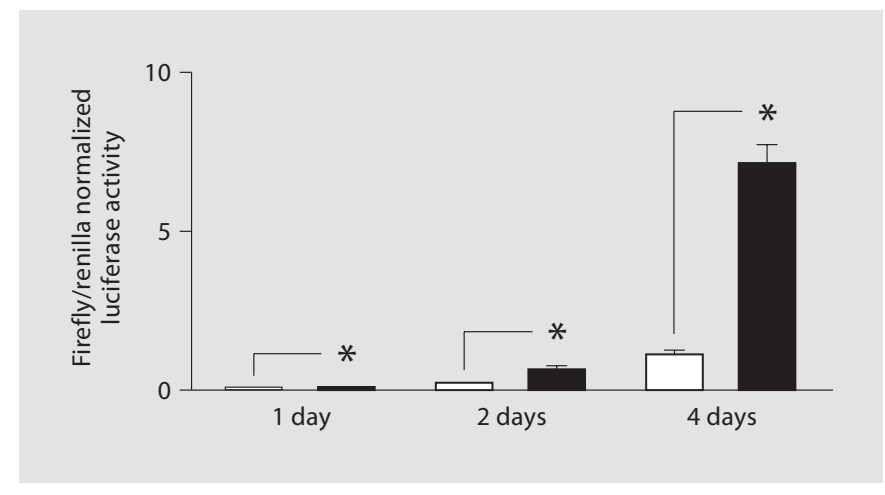

Fig. 1. PC-1 promoter activity increases with calvarial osteoblast differentiation. MC3T3E1(C4) cells were transfected with experimental PGL3/PC1 and control PGL4/hRluc luciferase constructs and grown in media containing no ascorbate $(\square)$ or media containing $50 \mu \mathrm{g} / \mathrm{ml}$ ascorbate ( $\boldsymbol{\square}$ ) for 1,2 or 4 days. Cell lysates were prepared and assayed for luciferase activity. Firefly luciferase activity is reported as normalized to renilla luciferase activity. Means \pm SD of 3 replicate experiments are shown. ${ }^{*} p<0.05$ versus culture without ascorbate.

mended protocol. Briefly, cells were incubated with DNA/Superfect complexes for $4 \mathrm{~h}$, in media containing $10 \%$ fetal bovine serum and penicillin/streptomycin. Cells were then washed with phosphate-buffered saline and medium was replaced. Cells were incubated for an additional $24 \mathrm{~h}$ after transfection prior to cytokine treatment and/or cell lysis.

\section{Cytokine and Inhibitor Treatments}

Cytokine treatments were conducted in media containing $0.5 \%$ fetal bovine serum and penicillin/streptomycin. Human recombinant FGF2 (Peprotech) was added to a final concentration of $50 \mathrm{ng} / \mathrm{ml}$ along with $1 \mu \mathrm{g} / \mathrm{ml}$ heparin (Sigma) and cells were incubated for $24 \mathrm{~h}$ prior to cell lysis for luciferase activity. Heparan sulfate proteoglycans, in the form of heparin or receptor heparin sulfate glycosaminoglycan modification, are required for high-affinity binding of FGF2 to the FGF receptor. Heparin was included in all FGF2 treatment experiments in order to eliminate the possibility of differential FGF2/receptor-binding affinity under different experimental conditions.

\section{Luciferase Assays}

Luciferase expression was assayed using the Dual Luciferase Assay System (Promega) and a Moonlight 2010 luminometer (Analytical Luminescence Laboratory), following the manufacturer's protocol. Cells were cotransfected with experimental firefly and control renilla luciferase constructs. After culture, cells were lysed and then centrifuged at $13,000 \mathrm{rpm}$ for $10 \mathrm{~min}$ at $4^{\circ} \mathrm{C}$. Supernatants were stored at $-80^{\circ} \mathrm{C}$ until use. Twenty-microliter aliquots of cell lysate were combined with $100 \mu$ l of luciferase assay substrate and $100 \mu \mathrm{l}$ of Dual glo (Promega) for measurement of firefly and renilla luciferase activity.

Osteoblastic Expression of PC-1

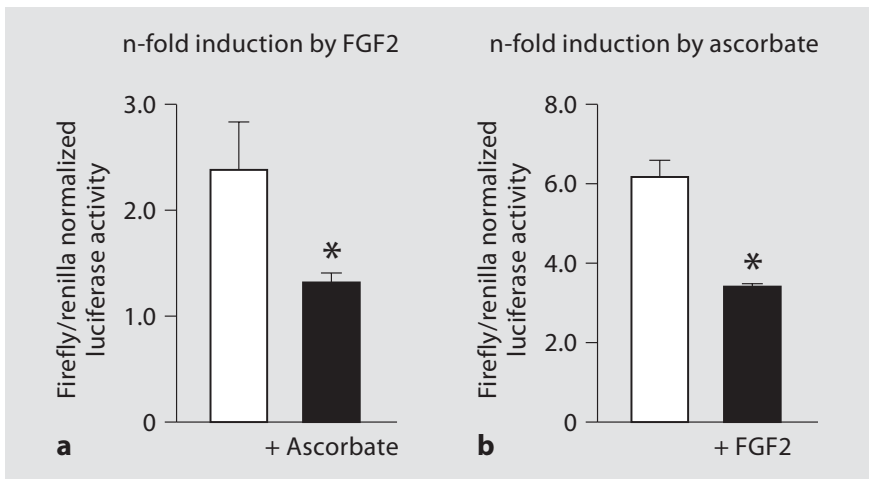

Fig. 2. PC-1 promoter responsiveness to FGF2 is inhibited by osteoblast differentiation and $\mathrm{PC}-1$ promoter activity induced by osteoblast differentiation is inhibited by FGF2. MC3T3E1(C4) cells were transfected with PGL3/PC1 and control PGL4/hRluc luciferase constructs and grown in media containing no ascorbate or media containing $50 \mu \mathrm{g} / \mathrm{ml}$ ascorbate for 4 days. Cells were then treated with $50 \mathrm{ng} / \mathrm{ml} \mathrm{FGF2}$ and cultured for an additional $24 \mathrm{~h}$. Cell lysates were prepared and assayed for luciferase activity. Firefly luciferase activity is reported as normalized to renilla luciferase activity. Means $\pm \mathrm{SD}$ of 3 replicate experiments are shown. $\mathbf{a}{ }^{*} \mathrm{p}<0.05$ versus no ascorbate. $\mathbf{b}{ }^{*} \mathrm{p}<0.05$ versus no FGF2 treatment.

\section{Results}

\section{PC-1 Promoter Activity Increases with Osteoblast Differentiation}

PC-1 expression increases with osteoblast differentiation at both mRNA and protein levels [Johnson et al., 1999]. In order to establish that activity of our PC-1 gene promoter/firefly luciferase reporter construct correlates with expected changes in gene expression, we examined PC-1 promoter activity in differentiating MC3T3E1(C4) calvarial osteoblastic cells. Results show that PC-1 promoter activity increased significantly with osteoblast differentiation, as induced by culture in media containing ascorbate (fig. 1). Of note, PC-1 promoter activity increased in culture containing ascorbate to a significantly greater extent than in culture containing no ascorbate. PC-1 promoter activity in cells cultured with ascorbate versus cells cultured without ascorbate was 1.7 times higher after 1 day, 2.3 times higher after 2 days and 6 times higher after 4 days of culture.

\section{PC-1 Promoter Responsiveness to FGF2 Diminishes with Osteoblast Differentiation}

In order to more directly study the mechanism by which PC-1 expression is regulated in preosteoblastic and osteoblastic cells, we have constructed a PC-1 gene pro- 


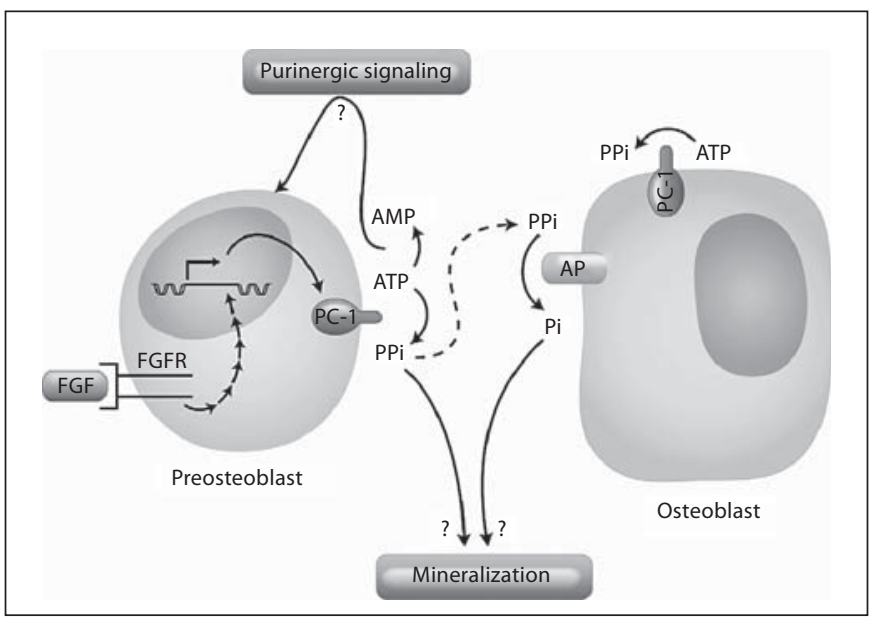

Fig. 3. Potential role of FGF2-induced preosteoblastic PC-1 expression in the development of mineralized tissues. FGF signaling in preosteoblastic cells induces expression of PC-1. PC-1 activity generates increased extracellular levels of inorganic pyrophosphate (PPi) via the hydrolysis of ATP. Extracellular pyrophosphate inhibits mineralization, but PC-1-generated pyrophosphate can also be hydrolyzed into inorganic phosphate $(\mathrm{Pi})$ by AP, which is expressed in more mature osteoblasts. Extracellular phosphate enhances bone mineralization. Alternatively, preosteoblastic PC-1 activity could affect preosteoblastic proliferation and/or differentiation via changes in purinergic receptor signaling, as PC-1 activity diminishes extracellular levels of ATP, a purinergic receptor ligand. FGFR $=$ FGF receptor; AMP = adenosine monophosphate.

moter/firefly luciferase reporter construct and shown that this reporter construct is selectively responsive to FGF2 in preosteoblastic cells [Hatch, 2007]. Because PC1 expression is also known to increase with osteoblast differentiation, here we have attempted to induce PC-1 promoter activity by FGF2 treatment in differentiating calvarial osteoblasts. Results show that FGF2 treatment induced PC-1 promoter activity to a significantly greater extent (2.4-fold increase) in undifferentiated cells than in differentiated cells (1.2-fold increase) (fig. 2a). Additionally, osteoblast differentiation by culture in media containing ascorbate was found to induce $\mathrm{PC}-1$ promoter activity in non-FGF2-treated cells to a significantly greater extent than in FGF2-treated cells (6-vs. 3 -fold increase in promoter activity) (fig. 2b).

\section{Discussion}

Results from studies investigating the effects of fibroblast growth factor signaling on bone mineralization indicate that FGFs may act to induce expression of factors that exhibit conflicting effects upon mineralization. Because pyrophosphate can inhibit or enhance bone mineralization, we hypothesized that FGF signaling would regulate expression of $\mathrm{PC}-1$, the primary enzymatic generator of pyrophosphate in osteoblastic cells. Because PC-1 is expressed in differentiated osteoblasts, yet FGF2 is unable to induce PC-1 expression in differentiated osteoblasts, we have initiated an investigation into the mechanism by which PC-1 expression is regulated in both preosteoblastic and osteoblastic cells.

Utilizing a PC-1 gene promoter/firefly luciferase reporter construct, here we confirm that PC-1 promoter activity increases with osteoblast differentiation. Of note, we find that PC-1 promoter activity increases within 2 days of culture under osteoblast differentiation conditions. These results are consistent with previous reports that PC-1 mRNA expression is first seen at 4 days after differentiation and that $\mathrm{PC}-1$ protein expression is first seen at 7 days after differentiation of calvarial osteoblastic cells in culture [Johnson et al., 1999]. The earlier detection of PC-1 promoter activity found here most likely reflects the increased sensitivity of promoter activity assays compared to assays for mRNA or protein.

Here, we also report that responsiveness of the PC-1 promoter to FGF2 is inhibited by osteoblast differentiation. This result is consistent with our previous finding that FGF2 was unable to induce detectable PC-1 mRNA when MC3T3E1(C4) cells were differentiated [Hatch et al., 2005]. Significantly, here we also report that responsiveness of the PC-1 promoter to osteoblast differentiation was inhibited by FGF2 treatment. The finding that PC-1 promoter responsiveness to FGF2 diminishes with osteoblastic differentiation and that PC-1 promoter responsiveness to osteoblast differentiation is inhibited by FGF2 treatment indicates that the mechanism for inducing PC-1 gene expression in osteoblastic precursor cells is distinct from the mechanism for inducing PC-1 gene expression in differentiated osteoblasts. Future studies will be needed to establish the precise mechanism for regulating PC-1 expression in preosteoblastic versus osteoblastic cells.

Finally, the fact that FGF2 induces PC-1 expression in preosteoblastic cells but not in differentiated osteoblasts suggests the possibility that the primary function of preosteoblastic PC-1 expression is distinct from that in differentiated osteoblasts. The primary function of PC-1 in differentiated osteoblasts is believed to be the generation of extracellular pyrophosphate for hydrolysis into phosphate by AP, which is also expressed in differentiated osteoblasts. The primary function of preosteoblastic PC-1 
could also be the generation of extracellular pyrophosphate. This pyrophosphate would temporarily inhibit bone mineralization until hydrolysis to phosphate by AP, which would be expressed in more differentiated cells. In this way, increased preosteoblastic generation of pyrophosphate by FGF2 could account for both the transient mineralization-inhibitory and overall bone anabolic effects of FGF2. Yet, PC-1 has pyrophosphatase and phosphodiesterase activities. PC-1 activity at the plasma membrane decreases extracellular levels of ATP and increases extracellular levels of adenosine monophosphate, therefore PC-1 activity could influence the preosteoblast phenotype by influencing purinergic receptor signaling (fig. 3). Purinergic signaling has long been known to modulate gene expression and the osteoblastic cell phenotype, and has more recently been associated with the bone remodeling response to mechanical loading [Li et al., 2005; Orriss et al., 2006]. The influence of PC-1 on purinergic signaling in osteoblastic precursor cells has yet to be investigated, but seeing as a primary activity of PC-1 is to break down extracellular nucleotides, it seems possible that some or all of effects of PC-1 on preosteoblastic gene expression may occur through changes in purinergic receptor signaling. Future experiments should allow us to more definitively establish the role of preosteoblastic PC-1 expression as that of generating increased levels of extracellular pyrophosphate and/or diminishing extracellular levels of ATP.

\section{References}

-Anderson, H.C., D. Harmey, N.P. Camacho, R. Garimella, J.B. Sipe, S. Tague, X. Bi, K. Johnson, R. Terkeltaub, J.L. Millan (2005) Sustained osteomalacia of long bones despite major improvement in other hypophosphatasia-related mineral deficits in tissue nonspecific alkaline phosphatase/nucleotide pyrophosphatase phosphodiesterase 1 double-deficient mice. Am J Pathol 166: 17111720.

Dunstan, C.R., R. Boyce, B.F. Boyce, I.R. Garrett, E. Izbicka, W.H. Burgess, G.R. Mundy (1999) Systemic administration of acidic fibroblast growth factor (FGF-1) prevents bone loss and increases new bone formation in ovariectomized rats. J Bone Miner Res 14: 953959.

Hatch, N.E. (2007) Potential role of PC-1 expression and pyrophosphate elaboration in the molecular etiology of the FGFR-associated craniosynostosis syndromes. Orthod Craniofac Res 10: 53-58.

Hatch, N.E., F. Nociti, E. Swanson, M. Bothwell, M. Somerman (2005) FGF2 alters expression of the pyrophosphate/phosphate regulating proteins, PC-1, ANK and TNAP, in the calvarial osteoblastic cell line, MC3T3E1(C4). Connect Tissue Res 46: 184-192.

-Hessle, L., K.A. Johnson, H.C. Anderson, S. Narisawa, A. Sali, J.W. Goding, R. Terkeltaub, J.L. Millan (2002) Tissue-nonspecific alkaline phosphatase and plasma cell membrane glycoprotein-1 are central antagonistic regulators of bone mineralization. Proc Natl Acad Sci USA 99: 9445-9449.
Johnson K., A. Moffa, Y. Chen, K. Prizker, J. Goding, R. Terkeltaub (1999) Matrix vesicle plasma cell membrane glycoprotein-1 regulates mineralization by murine osteoblastic MC3T3 cells. J Bone Min Res 14: 883-892.

Johnson, K., J. Godin, D. Van Etten, A. Sali, S. $\mathrm{Hu}$, D. Farley, H. Krug, L. Hessle, J.L. Millan, R. Terkeltaub (2003) Linked deficiencies in extracellular PPi and osteopontin mediate pathologic calcification associated with defective PC-1 and ANK expression. J Bone Min Res 18: 994-1004.

Johnson, K., K. Pritzker, J. Goding, R. Terkeltaub (2001) The nucleoside triphosphate pyrophosphohydrolase isozyme PC-1 directly promotes cartilage calcification through chondrocyte apoptosis and increased calcium precipitation by mineralizing vesicles. J Rheumatol 28: 2681-2691.

Li, J., D. Liu, H.Z. Ke, R.L. Duncan, C.H. Turner (2005) The P2X7 nucleotide receptor mediates skeletal mechanotransduction. J Biol Chem 280: 42952-42959.

Liang, H., S. Pun, T.J. Wronski (1999) Bone anabolic effects of basic fibroblast growth factor in ovariectomized rats. Endocrinology 140: 5780-5788.
Montero, A., Y. Okada, M. Tomita, M. Ito, H. Tsurukami, T. Nakamura, T. Doetschman, J.D. Coffin, M.M. Hurley (2000) Disruption of the fibroblast growth factor-2 gene results in decreased bone mass and bone formation. J Clin Invest 105: 1085-1093.

Orriss, I.R., G.E. Knight, S. Ranasinghe, G. Burnstock, T.R. Arnett (2006) Osteoblast responses to nucleotides increase during differentiation. Bone 39: 300-309.

Register, T.C., R.E. Wuthier (1985) Effect of pyrophosphate and two diphosphonates on 45Ca and 32Pi uptake and mineralization by matrix vesicle-enriched fractions and by hydroxyapatite. Bone 6: 307-312.

Sobue, T., T. Naganawa, L. Xiao, Y. Okada, Y. Tanaka, M. Ito, N. Okimoto, T. Nakamura, J.D. Coffin, M.M. Hurley (2005) Over-expression of fibroblast growth factor-2 causes defective bone mineralization and osteopenia in transgenic mice. J Cell Biochem 95: 83-94.

Terkeltaub R., M. Rosenbach, F. Fong, J. Goding (1994) Causal link between nucleotide pyrophosphohydrolase overactivity and increased intracellular inorganic pyrophosphate generation demonstrated by transfection of cultured fibroblasts and osteoblasts with plasma cell membrane glycoprotein-1. Relevance to calcium pyrophosphate dihydrate deposition disease. Arthritis Rheum 37: 934-941. 